\title{
MÁS ALLÁ DE OCCIDENTE. FRANKFURT, ENTRE ANTROPOLOGÍA E HISTORIA CULTURAL
}

\author{
LUCiAno ArCELLA* \\ doi:10.11144/Javeriana.uph33-66.fahc
}

\begin{abstract}
RESUMEN
A partir de las primeras décadas del siglo XX en Alemania se afirmó una ideología compartida que tuvo como base común el rechazo del racionalismo occidental. Este fue considerado un medio ineficaz para comprender tanto las civilizaciones antiguas (el mundo griego, de acuerdo a la concepción nietzscheana interpretada por W. F. Otto, y las civilizaciones monumentales en general estudiadas por Spengler) como las civilizaciones tradicionales o "primitivas" (estudiadas por Frobenius, investigador de las culturas africanas). Por eso se sintió la necesidad de llegar a una forma diferente de comprensión basada en capacidades intuitivas, en una participación emotiva. Sin embargo, para llegar a la aplicación concreta de esta perspectiva, para conseguir una comprensión efectiva del otro (o del "tú", como escribe Frobenius), se entendió que alguna manera había que "ser" el otro, entrar en él, dejarse "agarrar" (ergreifen) por su mundo renunciando a la centralidad del yo, occidental y tal vez humano.

Palabras clave: racionalismo; occidente; civilización; intuición; ergreifen
\end{abstract}

* Universidad del Valle, Cali, Colombia.

Correo electrónico: luciano.arcella90@gmail.com

Para citar este artículo: ARCELla, L. (2016). Más allá de occidente. Frankfurt, entre antropología e historia cultural. Universitas Philosophica, 33(66), pp. 217-236. ISSN 0120-5323, ISSN en línea: 2346-2426, doi:10.11144/Javeriana.uph33-66.fahc 


\title{
BEYOND THE WEST. FRANKFURT, BETWEEN ANTHROPOLOGY AND CULTURAL HISTORY
}

\author{
LuCiano ArCELla
}

\begin{abstract}
With the beginning of the $\mathrm{XX}^{\text {th }}$ century in Germany prevailed a common ideology based on the reject of Western rationalism. It was seen an ineffective instrument to understand ancient civilizations (Greek culture, according to Nietzsche's point of view that W. F. Otto assumed, and monumental civilizations researched by Spengler) and traditional or "primitive" civilizations (Frobenius, like researcher in African cultures). Therefore, one felt the need of a different form of understanding based on intuitive abilities, on an emotional involvement. However, in order to apply positively this view and to gain an effective awareness of the other (the "you", writes exactly Frobenius), one realized that it was necessary "to be" the other, to get into him, to be grabbed (ergreifen) by his world and abdicate the central role of the self, Western or maybe human.

Key words: rationalism; west; civilization; insight; ergreifen
\end{abstract}


A partir de los primeros años del siglo XX, la ciudad de Frankfurt am Main se volvió un centro de importantes fermentos culturales y tuvo un notable incremento de las instituciones académicas. Al mismo tiempo tuvo una expansión urbana con la que adquirió aquel carácter de centro internacional de comercio y de comunicaciones que hoy la acredita.

En el ámbito específico de los estudios antropológicos se señalan eventos significativos, como la abertura del Museum für Völkerkunde (Museo Etnológico) y la transferencia de Múnich del Forschungsinstitut für Kulturmorphologie fundado por Leo Frobenius en 1922. En la capital bávara el Instituto estaba ubicado en el castillo de Nymphenburg, mientras en Frankfurt fue hospedado en la Goethe Universität. En 1932 Frobenius fue nombrado docente honorario de etnología en esta universidad, y dos años después asumía el cargo de director del museo. Sus colaboradores más estrechos fueron Jensen ${ }^{1}$ y Niggemeyer ${ }^{2}$.

El Instituto evidenciaba, a través la riqueza de los materiales de sus archivos (sobre todo el Felsbilderearchiv, archivo de los grafitos rupestres) y la amplitud de sus iniciativas, la enorme capacidad operativa de su director ${ }^{3}$, y por eso se proponía como polo de referencia de la ciencia antropológica en aquel tiempo.

1 Adolf Ellegard Jensen (1899-1965), compartió con su maestro Frobenius la teoría de la Kulturmorphologie, y consideró como base del concepto religioso el principio de "dema", mutuado de la cultura Marind-Anim en Nueva Guinéa. Este pueblo consideraba los "dema” como divinidades originarias nacidas de los miembros esparcidos de Hainuwele, así como nacen los tubérculos plantados en el suelo.

2 H. Niggemeyer (1908-2005), fue asistente en el Frobenius Institut a partir del 1934 y participó en un proyecto de investigación en las Islas Molucas guiado por Jensen. Después de la guerra fue director del Museum für Weltkulturen.

3 Entre las importantes iniciativas de Frobenius, Vajda evidencia "die zwölf grössen Forschungsreise" y el monumental Atlas Africanus (Vajda, 1973, p. 21). 
Junto a los indicados colaboradores, participaban al curatorium científico del Instituto, los etnólogos Lommel ${ }^{4}$, Rhotert ${ }^{5}$ e Zerries ${ }^{6}$, y los clasicistas Reinhard ${ }^{7}$ y Otto.

Los intereses del Forschunginstitut, además, no se limitaban al sector de la ciencia antropológica, es decir, al estudio de las culturas tradicionales (aunque fue esto el sector específico al cual se dedicó el director), pero, por su declarado interés para la "morfología de las culturas", Frobenius abrió, en el ámbito del mismo Instituto, un centro de investigación para el estudio del mundo clásico. Esto estaba de acuerdo con la visión general de elaborar una Kulturgeschichte dirigida en la comprensión de las civilizaciones en su complejidad.

Prueba de este interés fue la adhesión al Instituto del citado clasicista Walter Friedrich Otto, de Oswald Spengler y de Franz Altheim, aunque los dos últimos, filósofos de la historia, no ocuparon ningún cargo oficial.

De esta comunión de intereses y de una convergencia ideológica surgió una investigación heterogénea (el campo de las culturas tradicionales para Frobenius, de las civilizaciones clásicas y modernas, respectivamente para Otto, Altheim y Spengler) pero fundada sobre una metodología y una ideología compartidas. Se trataba esencialmente de salir de la perspectiva de la cultura occidental, con el fin de entender otras expresiones culturales y participar "emotivamente" de sus valores.

Otto cumplió parte de sus estudios en la Universidad de Bonn, en donde tuvo como maestro a Hermann Usener, cuyo interés precipuo fue la relación entre antropología y die Antike, el mundo clásico, en cuanto consideraba la ciencia

4 Andreas Lommell (1912-2005), fue asistente científico del Frobenius Institut del 1937 al 1940. A partir de 1957 fue director del Staatliche Museum für Völkerkunde de Múnich

5 Hans Rhotert (1900-1991) fue un fiel discípulo de Frobenius y lo acompañó en algunas de sus expediciones en África. Alejado del Instituto Etnológico de Múnich después de la guerra, consiguió en el 1957 el cargo de director del Linden-Museum de antropología en Stuttgart.

6 Otto Zerries (1914-1999), estudioso de las culturas del Norte América, a partir del 1956 fue director del sector América del Staatliche Museum für Völkerkunde de Múnich y docente de etnología de la L. M. Universität de la misma ciudad.

7 Karl Reinhardt (1886-1958), filólogo y estudioso del mundo antiguo, fue docente en la Universidad de Frankfurt a partir del 1924, y después de la interrupción de la guerra, retomó su cargo en la década del sesenta. Hizo importantes estudios sobre Parménides, Sófocles y la concepción cósmica en la cultura griega. Este trabajo fue publicado con el título Kosmos und Sympathie (1926). 
histórica "Anthropologie in höherem Sinne". Después de la licenciatura, en 1897, pasó un tiempo en Múnich, donde tuvo un cargo en el Thesaurus Linguae Latinae y, como indica Cancik (1988, p. 72), frecuentó los cursos de psicodiagnóstica de Klages y estuvo en contacto con el grupo de los "Cósmicos" y con los círculos "gueorguianos". Docente en la Universidad de Frankfurt del 1914 al 1934, Otto se encontró con Frobenius, con quien adhirió al "Doorner Kreis", constituido en el 1918 en la pequeña ciudad de Holanda por el Emperador Wilhelm II, al fin de apoyar estudios de carácter antropológico.

En el tiempo de su docencia en Frankfurt, Otto fue editor de una serie de libros dedicados al estudio del mundo clásico, los Frankfurter Studien zur Religion und Kultur der Antike, serie editada por Vittorio Klostermann. Salieron dieciséis volúmenes; el primer título fue Mater Larum. Zum Wesen der Larenreligion, de Ernst Tabeling, publicado en 1932. El último libro de la serie fue el trabajo de Karl Schlechta, Goethe in seinem Verhältnis zur Aristoteles, publicado en 1938, cuando Otto ya era docente en la Universidad de Königsberg. En la misma serie fueron publicados Dionysos, Mythos und Kultus, de Otto, 1933, y los dos volúmenes del trabajo de Franz Altheim, Epochen der Römischen Geschichte (noveno y décimo tercero de la serie, editados respectivamente en 1934 y 1938). Altheim participó directamente, además, en las actividades del Instituto, porque estuvo con Frobenius en su expedición en Val Camonica para estudiar los grafitos rupestres, con la finalidad de demostrar la llegada de pueblos indo-europeos en la península itálica.

Otra figura que fue involucrada en la actividad, o más bien en la ideología del Instituto, fue el filólogo e histórico de las religiones Károly Kerényi, que

8 "Antropología en sentido más alto" (las traducciones del alemán al castellano son del autor de este artículo).

9 Stefan George (1868-1933) fue un poeta “visionario", en el sentido que consideraba la poesía expresión religiosa o ascética, de acuerdo con una visión del mundo esencialmente aristocrática. Por eso produjo el interés de Goebbels y de la dirigencia nacional-socialista, que le propuso el cargo de ministro de la cultura; cargo que él rechazó, así como, con la afirmación del nazismo en Alemania, decidió migrar a Suiza. Varios críticos consideran con estupor su rechazo de la ideología nazista a partir de su espíritu heroico, elitista, pero ignorando que el movimiento de la Konservative Revolution, que compartía los ideales aristocráticos de George, se reconoció solo parcialmente en la ideología hitleriana, porque la considerada populista y al mismo tiempo materialista, a partir de su visión de un racismo biológico y no espiritual. En relación con estos argumentos, véase: Mohler (2005) y Evola (2009). 
encontró a Otto en 1929, en el curso de una instancia en Atenas, donde el joven húngaro gozaba de una beca del Instituto Arqueológico local. Kerényi se podía considerar un prosélito de la escuela filológica de Wilamowitz, del cual había frecuentado las clases en la Universidad de Berlín. Después de haber conseguido la docencia libre en la Universidad de Budapest, consiguió en el 1936 la docencia de filología clásica en la Universidad de Pécs. Al tiempo de su encuentro con Otto, él ya podía darse el lujo de tener una rica producción científica, en la cual se evidenciaba el trabajo Die griechische-orientalische Romanliteratur in religionsgeschichtliecher Beleuchtung. EinVersuch (1927) ${ }^{10}$.

El encuentro con Otto y el siguiente contacto con Frobenius, fueron eventos decisivos en la formación de Kerényi, por cuanto representaron la finalización de una instancia específica: poder conjugar el estudio filológico con un estudio global de la cultura. Sin embargo, se puede notar que él no consideró este nuevo enfoque de sus estudios como algo realmente diferente en relación con lo que había aprendido de sus maestros -en contraste con Schlesier, que lo define "der Schulphilologie überdrussig” (1994, p. 265)- más bien, lo consideró como prosecución de un camino en el cual el mismo Wilamowitz lo había colocado ${ }^{11}$.

Si Frankfurt, entonces, pudo representar el lugar de encuentro entre etnología y filología en el ámbito de una concepción histórico-cultural, Alemania, al inicio del siglo XX, manifestó una tendencia general para acercar las dos disciplinas en nombre de una global Kulturgeschichte. Clara expresión de esta tendencia fue la revista Archiv für Religionswissenschaft, fundada por Thomas Achelis en el 1904. En esta publicación, que representaba Usener y la Escuela de Bonn, participaron, entre

10 De la novela helenística y de su mundo cultural se había ocupado Rohde (1876) y también Altheim (1951).

11 Un signo de su actitud en favor de Wilamowitz y su enseñanza, se encuentra en el ensayo Walter $F$. Otto zum achtzigsten Geburtstag (1954), en el cual Kerényi, citando la conferencia de su maestro, Philologie und Schulreform, evidencia como él evaluaba la "unidad" de la Kultur, y tenía como su precipua finalidad en entenderla en su globalidad. 
otros, Deubner ${ }^{12}$, Weinreich ${ }^{13}$, Norden ${ }^{14}$ y el sueco Nilsson ${ }^{15}$, que aplicaron sus estudios filológicos a la comprensión global de la cultura antigua.

En aquel tiempo, otro centro focal de la cultura etnológica alemana era representado por el grupo que se había constituido alrededor de Wilhelm Schmidt en Viena en 1932, y trasladado a Freiburg en 1938. Schmidt, junto con los etnólogos Graebner y Ankermann, asumía el concepto de Kulturkreis (esfera o área cultural) mencionado por Frobenius en el trabajo Der afrikanische Kulturkreis (1897) y elaborado en la obra sucesiva, Der Ursprung der Afrikanischen Kulturen (1898).

De todas maneras, el núcleo del cual se originaba un nuevo conocimiento, cuya finalidad era crear una síntesis entre filología, historia cultural y etnología, se podía individualizar, sobre todo, en la tríada Otto, Altheim, Frobenius, en cuanto intérpretes de un "conocimiento circular", por el cual las tres disciplinas se juntan en un estudio finalizado en la comprensión de las culturas con su propensión unitaria. Fue partícipe de esta visión también Oswald Spengler, que aunque no tuvo contactos directos con este grupo (Spengler no fue un académico), se apoderó de los elementos principales de la ideología de Frobenius, a partir de la concepción de la cultura en cuanto organismo con vida propia, y la consiguiente idea de la relatividad de sus categorías identificativas.

\section{La concepción del "otro"}

Frobenius, en LA ELABORACión de su sistema base para el desarrollo de su trabajo etnográfico, tuvo presente, antes de una evaluación de carácter epistemológico, una motivación de carácter profundamente ético. En una época en la cual

12 L.A. Deubner (1877-1946), estudioso de la cultura griega, fue docente en la Humboldt Universität de Berlín; entre sus trabajos, se destaca el título: Attische Feste (1932).

13 O. Weinreich (1886-1972), docente de filología clásica en la Universidad de Heidelberg. Autor de Versuchungen zum Wunderglauben der Griechen und Römer (1909).

14 E. Norden (1848-1941), cumplió su estudio universitario de filología en Bonn, participando al grupo de Usener. Sucesivamente, en cuanto judío, padeció el ostracismo nazista. En sus trabajos se ocupó precipuamente de la cultura romana.

15 M. P. Nilssohn (1874-1967), estudioso de la cultura griega, fue docente en la Universidad de Lund. Autor de Primitive Religion (1911). 
el abundante florecer de un interés histórico se traducía en trabajos que, tratando la historia mundial, se ocupaban, a partir del interés fundamental en Europa, de un área que podía llegar hasta Egipto y Asia Menor, los pueblos de África, Sudamérica y Oceanía eran considerados pertenecientes a un estado de naturaleza, o también como grados inferiores de una "Evolución unívoca (...) en cuyo escalón más alto fue colocada la civilización europea" ${ }^{16}$ (Vajda, 1973, p. 28). Frobenius sintió la exigencia de rescatar la dignidad del continente africano y de un pueblo "cuya cruda depravación no había producido otra cosa que el fetichismo"17 (Vajda, 1973, p. 29). Por lo tanto, como indica todavía Vajda, Frobenius tuvo que producir una verdadera revolución copernicana en el Weltbild de los Europeos, para afirmar el principio de que "los pueblos de África y de los otros continentes extráenos (...) tienen una propia historia” 18 (Vajda, 1973, p. 29).

Para llegar a esta visión, era necesario, ante todo, superar el etnocentrismo radical basado en el prejuicio de la evolución unilineal de la civilización humana y, con ello, elaborar una metodología por la cual la observación pudiera liberarse de la mirada (cultural y "moral") por parte del observador. Es así que el principio de la especificidad de cada civilización, concepto significativo de la Kulturkreislehre, representó una objeción radical a la idea de la linealidad, que había alimentado la filosofía de la historia hegeliana, junto a una concepción "popular" que se había traducido en la praxis política de la colonización europea del continente africano. Es decir que, aunque la doctrina de las esferas culturales no nace como directa confutación del principio de evolución representa, sin embargo, la voluntad de entender diferentes expresiones culturales por sus especificidades, sin la obligación de ordenarlas en un sistema jerárquico.

En relación con el carácter "vivo" („das lebendige“) de la cultura, Frobenius (1898) considera que la misma, en cuanto "organismo vivo tiene un nacimiento, una infancia, una edad adulta, una vejez y al fin una destrucción”19 (p. X). Es la

16 „...einbahn-Evolution ... auf deren höchste Stufe die europäische Kultur gesetzt wurde“.

17 „... dessen rohe Verkommenheit nur den Fetischismus produziert hatte und sonst nichts".

18 ,...die Völker Afrikas und der anderen fremden Kontinente [...] eine eigene Geschichte haben“.

19 „....ebendiger Organismus, erlebe also eine Geburt, eine Kinder-, Mannes- und Greisenalter und endlich ein Hinscheiden". 
misma idea que poco tiempo después fue elaboraba por Spengler (1923), para quien la civilización:

... tiene nuevas posibilidades de expresión, que aparecen, maduran, mueren, no vuelven más. Hay artes plásticas, pinturas, matemáticas, físicas, completamente diferentes entre ellas, cerradas en sí mismas con una duración limitada (...). Estas civilizaciones, expresiones vitales de alto nivel, crecen en la falta total de fines, como las flores en los campos. (...) Veo en la historia del mundo el cuadro de una eterna composición y descomposición, un formarse y un descomponerse de formas orgánicas. (p. 29) ${ }^{20}$

Además, escribe Frobenius (1958): "La civilización es un organismo, vida social y familiar, intuición del mundo y religión, arte y literatura son expresiones de esta unidad"21 (p. 70).

Así mismo, el concepto de organismo pertenece al de unidad; por lo cual, no se puede estudiar una sociedad separándola en sus elementos: ella no puede ser comprendida a través del análisis de elementos de la cultura material ("Dingen der materiellen Kultur"), sino solo en su forma global, que se expresa en un principio que la transciende. En este sentido, es fundamental colocar en primer plano los comportamientos, las actitudes, los sueños, las fantasías y los mitos.

Entonces, en relación con ciclo vital de las culturas, por lo que concierne a la fase inicial o infantil, Frobenius indica una específica caracterización, que él define con el término Ergriffenheit, en cuanto "capacidad de ser agarrado por la esencia de los hechos"22 (Frobenius, 1933, p. 25). En esta fase el individuo, así como una cultura, vive una relación particular con el ambiente $(\text { Umwelt })^{23}$, con

20 „...hat ihre neuen Möglichkeiten des Ausdrucks, die erscheinen, reifen, verwelken und nie wiederkehre. Es gibt viele, im tiefsten Wesen völlig voneinander verschiedene Plastiken, Malereien, Mathematikern, Physiken, jede von begrenzter Lebensdauer, jede in sich selbst geschlossen [...] Diese Kulturen, Lebewesen höchsten Ranges, wachsen in einer erhabenen Zwecklosigkeit auf, wie di Blumen auf dem Felde. [...] Ich sehe in der Weltgeschichte das Bild einer ewigen Gestaltung und Umgestaltung, eines wunderbaren Werdens und Vergehens organischer Formen".

21 "La civiltà è un organismo. Vita sociale e familiare, intuizione del mondo e veligione, arte e letteratura sono espressioni di questa unitä" (frase citada de la edición italiana de Kulturgeschichte Afrikas, traducción del autor del artículo).

22 „Fähigkeit ergriffen zu werden vom Wesen der Tatsachen“.

23 Spengler substituyó el término "Umwelt" (ambiente) con "Landschaft" (paisaje). De todas maneras, los dos términos no se limitan a la sola realidad material. 
el cual se siente en íntima conexión. A propósito de esto, M. F. Chevron (2004) escribe: "El primer escalón del desarrollo cultural muestra, en cuanto se lo describe como un estadio, donde se encuentra la inmediata percepción del ambiente y de la realidad"24 (p. 165). Esta actitud, si por un lado puede indicar una posición pasiva del sujeto en frente al objeto, al mismo tiempo evidenciaría, según Jensen (1938/1940), su fuerza creadora, ya que "con la «Ergriffenheit» se indicaría aquella sola condición que permite al hombre un acto creativo"25 (p. 55).

Aquí encontramos una divergencia de opiniones acerca de la posición del hombre en relación con el ambiente; es decir, si el ser humano resulta pasivo o si es el verdadero protagonista, porque, asimilando lo que está en sus alrededores, lo transforma de manera creadora. Jensen coloca en relieve la función activa del hombre con base en la voluntad de atribuirle un papel de protagonista, pero de tal manera no evalúa el elemento clave de la teoría de Frobenius, por el cual no son los individuos con sus voluntades los que pueden elaborar un complejo cultural, sino que es este, operando como sujeto, el que se impone y no deja libertad de elección.

Como observa Chevron (2004), "el punto central es el dominio del ambiente en cuanto ordena toda la vida y el pensamiento de los seres humanos en una civilización" ${ }^{26}$ (p. 165). Para sustentar su tesis, cita una enunciación del mismo Jensen, tal vez parcialmente contradictoria en relación con la expuesta arriba: "No solo el ser humano es el acto creativo de otro, más bien, también su ambiente, que lo agarra, se vuelve en este sentido, otro"27 (Jensen, 1938, p. 55).

Dejando de lado las fases sucesivas del desarrollo de las civilizaciones, que Frobenius caracteriza como Ausdruck (fase madura) y Anwendung (fase terminal), correspondientes a la racionalización y a la instrumentalización, consideramos la primare fase de este proceso y el dato fundamental de la "pasividad" del ser

24 ,...die erste Stufe der kulturellen Entwicklung darstellt, als ein Stadium beschrieben wird, auf welchem die unmittelbare Wabrnehmung der Umwelt und der Wirklichkeit stattfindet".

25 „Mit Ergriffenheit sollte eben jener Zustand bezeichnet werden, der allein den Menschen zu eine schöpferischen Akt befähigt".

26 „Ausgangspunkt ist die angenommene Dominanz einer Erscheinung der Umwelt, die das ganze Leben und Denken der Menschen in einer Kultur regelt". 
humano en frente a una alteridad representada por una Umwelt que solo parcialmente puede identificarse con la naturaleza, en cuanto se trata de un contexto cósmico, de un conjunto de elementos por los cuales el alma receptiva del sujeto (el individuo como la cultura) es penetrada.

Así es que la pasividad del ser humano en la dialéctica de Frobenius, consigue un significado fundamental, en la medida en que este desarrollo dialéctico no parte del yo, de su inicial aislamiento, para acabar en una síntesis en la cual el otro resulta funcional a la afirmación del sujeto, pero de una alteridad cósmica que se impone por su fuerza. Y aunque no acaba con la afirmación final del sujeto, sí lo hace con su extinción (el fin natural de las civilizaciones).

La modalidad cognitiva y formativa de la fase originaria de la civilización es elaborada ulteriormente en la obra de carácter más específicamente teórico: Paideuma. El término, asumido del Timeo platónico -kathaper eikos ghennemata kai paideumata theon ontas - indica, para Frobenius, la predisposición de aprender por parte de un grupo, que acoge algo que se impone por su fuerza persuasiva. Esto indica, entonces, que una civilización se forma a partir de una fuerza externa, a la cual responde la fuerza reactiva del sujeto, que determina a sí mismo a través de esta mediación ${ }^{28}$.

De esta manera, la formación del individuo como de una civilización parte de un elemento externo, que siempre se impone a quien no puede evitar de aceptarlo. Con la consecuencia de que una cultura se afirma en su conjunto, en aquella Ganzheit que expresa su único e inconfundible "estilo". Esto quiere decir que cada elemento que forma una cultura no puede ser entendido aisladamente, sino que cada concepto, cada palabra, consigue su auténtico significado exclusivamente en un contexto cultural.

Esta concepción la confirma Spengler (1923) al declarar:

28 Haciendo una referencia al pensamiento platónico, notamos cómo, a diferencia de la dialéctica "socrática", que parte del sujeto que busca la verdad en la confrontación con el otro, en la que se puede definir "técnica dialéctica" utilizada por su discípulo, el procedimiento empieza a partir del otro, del "minister", que con sus preguntas hace de manera que la verdad surja en el sujeto. Especificamos que para la distinción entre el Sócrates originario, es decir la fase en que Platón se limitaba en relatar fielmente el pensamiento del maestro, y la fase en que Platón, aunque utilizando como protagonista de los diálogos la figura de Sócrates, elaboraba su filosofía con su diferente forma dialógica, se vea G. Giannantoni, Dialogo socrático e nascita della dialettica nella filosofía di Platone, Napoli, Bibliopolis, 2005. 
Todas las palabras básicas, como masa, substancia, materia, cosa, cuerpo, extensión y las millares de expresiones verbales correspondientes en las lenguas de otras civilizaciones, son sin duda alguna, signos determinados por el destino, que del infinito complejo de posibilidades, trae sus específicos significados solo en el ámbito de su propia civilización. Ninguna expresión puede ser traducida exactamente en la experiencia y en el conocimiento de otra civilización. Ninguna de estas palabras fundamentales puede volver una segunda vez. ${ }^{29}$ (p. 235)

Por lo tanto, en la identificación de los dos complejos culturales africanos fundamentales, la civilización etiópica y la camítica, Frobenius afirma que ellas se caracterizan por dos estilos cabalmente opuestos. Mientras la civilización camítica revela una peculiar severidad, la etiópica se caracteriza por su humor, su alegría (Frobenius, 1958, p. 539). En este sentido, se puede hablar de "estilo" de una cultura, en cuanto expresión de su estructura unitaria, que se impone a la percepción por su evidencia como Gestalt (figura, configuración).

La civilización, como afirma Frobenius (1921), es un "metafisischer es" (p. 58), que se impone al individuo como necesidad y destino. Principio que Spengler afirma con el notorio lema que acompaña su obra fundamental: "Ducunta fata volentem, nolentem trabunt".

Esto quiere decir que, puesto que una cultura, así como el individuo, se forma por medio de una voluntad autónoma del ser, que decide manifestar espontáneamente su esencia, la única forma de su conocimiento auténtico consiste en penetrar esta esencia. Por lo tanto, se necesita de una actitud intuitiva, que Frobenius (1921) define con el término "Einfüblung"(p. 57); así, para conseguir una efectiva comprensión, "el investigador intuitivo por lo contrario busca el ámbito delo que es vivo, en su falta total de reglas, al fin de compartir las emociones del alma"30 (Frobenius, 1921, p. 81).

29 „Alle Grundworte wie Masse, Substanz, Materie, Ding, Körper, Ausdehnung und die tausende in den Sprachen anderer Kulturen aufbewahrten Wortzeichen entsprechender Art sind Wabllose, vom Schicksal bestimmte Zeichen, welche aus der unendliche Fülle von Weltmöglichkeiten im Namen der einzelnen Kultur die einzig bedeutende und deshalb notwendige herausheben. Keines ist in das Erleben und Erkennen einer andern Kultur genau übertragbar, Keines dieser Urworte kehrt nochmals wieder". mitzuerleben". 
Este "mitleben", cual íntima participación al ser de una cultura, en los términos spenglerianos se traduce en el principio del conocimiento fisionómico, en oposición al conocimiento sistemático. En palabras de Spengler (1923): “Todas las maneras para comprender el mundo al fin pueden ser indicados como morfología. La morfología de lo mecánico y de lo extenso, es una ciencia que descubre y ordena a las leyes naturales y las relaciones causales, se llama sistemática. La morfología del orgánico, de la historia y de la vida, todo lo que tiene en sí una dirección y un destino, se llama fisiognómica"31 (p. 136).

Más allá de variaciones terminológicas que se refieren a los medios de acercamiento a una cultura, hay un principio fundamental común, que consiste en el carácter intuitivo del conocimiento, el cual responde al procedimiento de formación de la cultura misma. Esta no es el resultado de elecciones individuales y tampoco de grupos, más bien los individuos y sus elecciones son su producto en cuanto entidad metafísica. Por eso el conocimiento de una cultura no debe partir de sus elementos, sino de aquella voluntad originaria que los produjo, y de la cual ellos resultan síntomas.

Como el estado originario de la formación de una civilización tiene carácter receptivo (todavía es presente el principio de la Ergriffenheit), un conocimiento auténtico debe proceder del "tú", o sea del otro, del cual el yo depende en su determinación y presencia. Al respecto, Frobenius (1958) afirma que "para comprender realmente una obra (...) es necesario que el observador pueda y sepa renunciar a su propia orientación y aceptar la del creador. Solo si todos los pueblos aprenden a pensar en el «Tú » se puede conseguir el ideal humano de la civilización”32 (p. 48).

Esta actitud de "modestia", por lo que concierne la dependencia del yo del dato, del otro, representó una efectiva revolución en la cultura occidental y constituyó la base teorética que dio la posibilidad a Frobenius de acercarse a las culturas africanas, superando el etnocentrismo de su época, hasta considerar el valor

31 „Alle Arten, die Welt zu begreifen, dürfen Letzen Endes als Morphologie bezeichnet werden. Die Morphologie des Mechanischen und Ausgedehnten, eine Wissenschaft, die Naturgesetze und Kausalbeziehungen entdeckt und ordnet, heißt Systematik. Die Morphologie des Organischen, der Geschichte und des Labens, alles dessen, was Richtung und Schicksal in sich trägt, heißt Physiognomik".

32 "Per comprendere veramente un'opera [...] bisogna che l'osservatore possa e sappia rinunciare al proprio orientamento e accettare quello del creatore. Soltanto se tutti i popoli imparano a pensare nel 'Tu', può venir raggiunto l'ideale umano della civiltà". 
superior de varios pueblos africanos, de acuerdo con un metro construido sobre la dignidad y el respecto humano (Frobenius, 1958).

Walter F. Otto: la "figura" de los dioses

Walter F. OtTo, EN SU BREVE ensayo contenido en el texto de 1933 dedicado a Frobenius, evidencia lo que él considera fundamental de su concepción: la oposición al "mecanicismo" y al "más rígido racionalismo", a fin de afirmar un conocimiento de carácter intuitivo. Para él, la intuición "se diferencia del conocido método racional, que separa su objeto en las partes más pequeñas con el fin de comprender su constitución global por medio de una ordenada composición de todas las fuerzas. Su mirada, por lo contrario, se dirige a la totalidad del objeto, y en la concepción viva de la totalidad quiere reconocer a aquel ser específico que debe ser definido como su esencia”33 (Otto, en VV. AA., 1933, p. 9).

Otto, por lo tanto, rebate que la civilización no es la suma de propiedades y funciones de los individuos que la componen, sino que es, como él mismo afirma, una especie de "drittes Reich", que va más allá del orgánico y del inorgánico, en cuanto "Erscheinungsform der Natur", así que los individuos son sus "Trägern und Funktionären” (Otto, en VV. AA, 1933, p. 9). Naturaleza que en parte se traduce en ambiente natural pero que, sin embargo, es también fuerza cósmica creadora, que se manifiesta parcialmente a través la historia humana. Con estas consideraciones, Otto no expone exclusivamente el pensamiento de Frobenius, pero muestra su adhesión a la metodología del etnólogo, de la cual además se sirve para enfrentar sus estudios histórico-religiosos relativos al mundo antiguo, con la conciencia que no se puede lograr conocimiento del pasado sin penetrar en una diversidad difícilmente traducible en los términos y los valores del mundo cultural del observador.

En tiempos anteriores, Nietzsche evidenció con extrema fuerza cómo el producto más religioso que literario de la tragedia no podía ser explicado sin

33 ,...unterscheidet sich durchaus von der bekannten rationalen Methode, die ibren Gegenstand in seine kleinsten Teile zerlegt, um seine gesamt Beschaffenheit hermacht aus den gesetzmäßigen Zusammenwirken aller Einzelkräften zu verstehen. Ihr Blick dagegen ist auf das Ganze des Gegenstandes gerichtet und will in der lebendigen Anschauung der Totalität jenes eigentümliche Sein erkennen, das als sein Wesen bezeichnet werden muss". 
proyectarse en la radical diversidad del espíritu griego. Igualmente, Otto se dirigía a la religión griega con la premisa de cuanto fuera difícil entenderla por parte del Occidente contemporáneo, que tenía como modelo de religión el cristianooriental ${ }^{34}$. Coherentemente con este principio, Otto cumple un esfuerzo lingüístico (elaboración de expresiones que puedan penetrar intuitivamente su objeto y no de forma analítico-descriptiva) ${ }^{35}$, para evidenciar la especificidad de una religión que, en su íntima relación con la naturaleza, rechazaba todas prejudiciales éticas (Otto, 1968).

En la base de su metodología se encuentra el concepto de Gestalt, ampliamente tratado en su colección de ensayos, titulada Das Gestalt und das Sein (Otto, 1959). En síntesis, él define la expresión Gestalt, como "un fenómeno originario que así como la belleza no se deja determinar conceptualmente"36 (Otto, 1959, p. 82), y añade: "resulta claro que el ser que quiere revelarse en la figura es el divino"37 (Otto, 1959, p. 82).

Ante todo, esta figura o configuración se presenta como una manifestación, como algo "sensitivo", que concierne la vista o el oído, y que de todas maneras no pertenece a la lógica discursiva. Por él el mundo se manifiesta "como figura viva (...) como el ser de la cosa misma"38 (Otto, 1959, p. 78), y solo el hombre poético tiene la capacidad de revelar su esencia.

34 Otto elabora este concepto en Der Geist der Antike und die christliche Welt (1923).

35 En conexión con el carácter emotivo y evocativo del lenguaje de Otto, Cancik encuentra la prueba de la tendencia "irracionalista" del filólogo. "Nei suddetti capitoli - escribe Cancik en relación al Dionisos de Otto - si tende ad evitare una terminologia di carattere scientifico, per offrire belle parole arcaizzanti predilette dai Tedeschi: patria, rivelazione, destino" y nota cómo se quiere expresar la contradictoria esencia de Dionisos por medio de la "eliminazione dei principi logici di identità e di contraddizione" (Cancik, 1988, p. 63).

Con estas consideraciones Cancik refiere este "irracionalismo" del lenguaje al irracionalismo de fondo originado a partir de la fuente nietzscheana y alimentada con la frecuencia de los círculos gueorguianos e klagesianos. Pero, no considera en la tentativa, por parte de Otto, de encontrar nuevas expresiones lingüísticas, la voluntad de penetrar una realidad diferente, que el racionalismo del lenguaje tradicional no puede expresar.

36 „...ein Urphänomen, das sich ebenso wenig wie die Schönheit begrifflich bestimmen lässt“.

37 „Da wird es offenbar, dass das Sein, das sich in der Gestalt erschließen will, das Göttliche ist". „....als lebendige Gestalt... [como] das Sein der Dinge selbst“. 
En relación con la existencia del mito, Moretti va más allá al afirmar que este "es el espacio de la revelación del divino (...) el dios se revela al hombre para que él pueda verlo y ser agarrado. El espacio de la experiencia del encuentro con el divino es (...) el espacio específico del mito, y del mito en forma de palabra, la poesía" ${ }^{39}$ (Moretti, 1991, p. 299). Encontramos extremamente apropiada la expresión "esserne afferrato" (ser agarrado), mutuada de Frobenius, que reproduce, en el ámbito del pensamiento de Otto, la condición humana delante de la manifestación de algo que, a su presentarse se impone, de la misma manera del "extranjero" Dionisio en su capacidad de producir la posesión.

La Gestalt, además, en términos de la ciencia psicológica, indica la figura, la configuración, la forma, que en cuanto conjunto estructurado, precede las partes que lo componen y que se impone al observador, determinando a priori el contenido de su percepción. En 1890, en la revista científico-filosófica Vierteljahrsschrift für Wissenschaftliche Philosophie, Christian von Ehrenfels publicó el artículo titulado „Über die Gestaltqualitäten”, en que hacía referencia al escrito de Ernst Mach, „Beiträge zur Analyse der Empfindungen” (1886). En este ensayo, Mach sostiene que las "figuras espaciales y las figuras musicales o melodías se puedan «percibir» de manera inmediata" ${ }^{\prime 0}$ (citado de von Erenfels, 1890, p. 249). Con base en esta afirmación, von Ehrenfels se pregunta si la configuración espacial o la melodía son "un mero conjunto de elementos o algo nuevo en relación a estos, que queda ajeno al conjunto mismo" ${ }^{\text {”11 }}$ (von Ehrenfels, 1890, p. 250). La respuesta es que se trata de "algo nuevo y de alguna manera autónomo"42 (von Ehrenfels, 1890, p. 250). Así, el autor demuestra en su investigación que la totalidad, la figura espacial o musical, son algo diferente de la suma de sus componentes - "de eso resulta que las figuras son otro que la suma de los elementos” ${ }^{\text {”3 }}$ (Ehrenfels, 1890,

39 “... è lo spazio della rivelazione del divino (...) Il Dio appare all'uomo perché questi può vederlo ed esserne afferrato. Lo spazio dell'esperienza dell'incontro con il divino è (...) proprio lo spazio del mito, $e$ del mito in forma di parola: la poesia".

40 "Raumgestalten und selbst Tongestalten oder Melodien unmittelbar zu 'empfinden' vermögen“.

41 „eine bloße Zusammenfassung von Elementen, oder etwas diesen gegenüber Neues, welches zwar mit jener Zusammenfassung, oder doch unterschreibar von ibr vorliegt".

42 „...etwas Neues und bis zu gewissem Grade Selbstständiges“.

43 „Es muss daher jene Gestalten auch etwas Anderes sein, als die Summe der Elemente“. 
p. 260)-, es decir, existen Gestaltqualitäen que preceden o determinan todas la formas de percepción: visual, auditiva, táctil, etc.

Fue muy amplio el campo de investigación que abrió esta concepción que, además, representó la base de las sucesivas teorías psicológicas de Wertheimer (la Gestalt Psychologie) y de la Escuela de Berlín, en cuyo ámbito Köhler fundaba la revista Psychologische Forschung. Casual, pero no sin un emblemático significado, que Wertheimer empezase a llevar sus experimentos sobre la vista y el movimiento en Frankfurt (Experimentelle Studien über das Sehen von Bewegung, 1912) porque, aunque no se evidencien relaciones directas entre los diferentes ámbitos de estudio (psicológico, histórico-cultural, etnológico), por lo que se refiere a la figura, en cuanto imagen preexistente y totalizante (resaliendo a los orígenes del concepto, se podrían considerar Goethe y Herbart $\left.{ }^{44}\right)$, parece evidente la tendencia, por parte de las diferentes disciplinas y elaboraciones de pensamiento, a la común oposición a un conocimiento analítico en función de una diferente relación del sujeto con el dato, que impone su "transcendente unidad".

Esta concepción no se resuelve en posiciones subjetivistas, sino que, gracias a la facultad de la intuición, configura la manera como el individuo puede coger o recoger, en el sentido de acoger, en su pasividad creadora, la originaria estructura del Ser, prescindiendo de la evanescencia de sus manifestaciones. Es decir, el individuo puede llegar a la unidad de lo real, independientemente de la multiplicidad de sus signos (entendiéndolos como síntomas y no como causas) en cuanto esencia originaria.

Esta unidad cobija bajo el mismo signo, más allá de la ciudad de Frankfurt, sede común de este pensamiento, un cuadro del mundo unitario, que tiene su base en la filosofía de Nietzsche, que rechaza el prejuicio de la centralidad del hombre occidental o, todavía más, del hombre en general, en cuanto excepción en la historia del universo.

Esta concepción que Nietzsche utilizó a partir de su estudio sobre el mundo griego y su visión trágica, por la cual no se podía entender el arte trágico sin entender el espíritu de aquel pueblo, y sin conocer su música y su propensión estática, enfatizada en todo el curso del desarrollo de su filosofía, determinó un

44 Véase, en relación al origen de esta concepción: Poggi, 1994. 
profundo impacto sobre la cultura del siglo XX (como dice Gottfried Benn: todo lo que fue elaborado en el novecientos fue exegesis nietzscheana), que se manifestó en campos diferentes. La filología, a partir de la necesidad de superar el racionalismo de la cultura actual, se propuso, en particular con Walter Friedrich Otto, entender el dionisismo por medio de una participación emotiva y, por lo tanto, a través de un lenguaje que pudiera responder a una representación intuitiva de su objeto. Esta concepción muestra, una vez más, una herencia nietzscheana, de acuerdo con la importancia que el filósofo dio al estilo en todo el desarrollo de su filosofía ${ }^{45}$.

Fue un contacto directo y al mismo tiempo una convergencia ideológica derivada de un sentimiento común, lo que produjo el vivo intercambio entre los diferentes sectores disciplinarios de filología, etnología e historia, de acuerdo con la idea común de superar el racionalismo etnocéntrico de la modernidad occidental, con el fin de entender civilizaciones tradicionales (Frobenius) como las civilizaciones monumentales (Spengler). La nueva actitud y, en consecuencia, la nueva metodología, superficialmente consideradas "irracionalistas", evidenciaron, por el contrario, el límite del racionalismo occidental (hegeliano en su esencia) y la consecuente incapacidad de comprender otras expresiones de la cultura humana: civilizaciones del tiempo pasado como un mundo "prejudicialmente" declarado "primitivo".

En definitiva, en este coherente cuadro del mundo resulta fundamental la actitud "ética" del yo frente a la realidad del otro, ya se trate del antiguo mundo griego o de las culturas tradicionales africanas. Una eticidad que no parte del yo y no se concluye con su afirmación o confirmación, sino que parte del "tú" para concluirse con la modestia de la cíclica consumación del yo, en cuanto individuo pero, sobre todo, en cuanto civilización basada en una libre actividad del Ser que prescinde de la soberbia pretensión de centralidad de una humanidad (¿en general o la occidental?) que piensa representar su último sentido.

45 Escribe Nietzsche alrededor del estilo: „Ich sage zugleich nach allgemeinem Wort über meine Kunst des Stils. Einen Zustand, eine innere Spannung von Pathos durch Zeichen, eingerechnet des Tempo dieser Zeichen, mitzuteilen - das ist der Sinn jedes Stils ..."(Ecce Homo, KSA 6, 304s). "Hablo con palabras sencillas alrededor de mi arte del estilo. Compartir un estado, una íntima tensión emocional, con base en el ritmo de estos signos, es éste el sentido de todo estilo". 
Referencias

Altheim, F. (1951). Roman und Dekadenz. Tübingen: Niemeyer.

Arcella, L., (1999). Le ragioni di Francoforte. VV.AA. Károly Kerényi: incontro con il divino (141-157). Roma: Settimo Sigillo.

Cancik, H. (1988). Dioniso in Germania. Da Heinrich Heine a Walter F. Otto: una revisione di cent'anni. Roma. Rari Nantes.

Chevron,M.F.(2004).Anpassungund EntwicklunginEvolutionundKulturwandel: Erkenntnisse aus der Wissenschaftsgeschichte für die Forschung der Gegenwart und eine Erinnerung an das Werk A. Bastians. Munster: LIT Verlag.

Evola, J. (2009), Il mito del sangue, Padova: Edizioni di Ar.

Fischer, H., Ed., (1990). Völkerkunde im National-Sozialismus. Aspekte der Anpassung. Affinität und Behauptung einer wissenschaftliche Disziplin. Berlin: Dietrich Reimer Verlag.

Flashar, H. Ed. (1995). Altertumswissenschaft in den Jahren. Neue Frage und Impulse, Stuttgart: Franz Steiner Verlag.

Frobenius, L. (1897), Der westafrikanische Kulturkreis, Geographische Mitteilungen, Heft X und XI.

Frobenius, L. (1898). Der Ursprung der afrikanischen Kulturen. Berlin: Gebrüder Borntraeger.

Frobenius, L. (1921). Paideuma. Umrisse einer Kultur-und Seelenlehre. München: Beck.

Frobenius, L. (1958). Storia delle civiltà africane. Torino: Boringhieri.

Giannantoni, G. (2005), Dialogo socratico e nascita della dialettica nella filosofia di Platone. Napoli: Bibliopolis.

Jensen, A.E. (1938-1940). Leo Frobenius, Leben und Werk, Paideuma, I, pp. $45-58$

Kerényi, K. (1962). Die griechisch-orientalische Romanliteratur in religionsgeschichtlicher Beleuchtung. Darmstadt: Wissenschaftliche Buchgesellschaft.

Kerényi, K. (1954). Walter F. Otto zum achtziger Geburtstag. Paideuma, Band VI, Heft 1, pp. 1-15.

Mohler, A. - K. Wassermann, (2005). Die konservative Revolution in Deutschland 1918-1932: Ein Handbuch. Grasz: A. Ares. 
Moretti, G. (1991). La perduta prossimità di Dio. Mito e poesia in Walter Otto. Sudi Filosofici, 14-15, pp. 299-306.

Losemann, V. (1977). Nationalsozialismus und Antike. Studien zur Entwicklung des Faches Alte Geschichte 1933-45. Hamburg: Hoffmann und Kampe.

Nietzsche, W.F. (1967 ss.), Kritische Studienausgabe herausgegeben von G. Colli und M. Montinari. Berlin: de Gruyter.

Otto, W. F. (1923), Der Geist der Antike und die Christliche Welt, Bonn: F. Cohen.

Otto, W.F.(1933).Dionysos. Mythosund Kultus. Frankfurt: Vittorio Klostermann.

Otto, W.F. (1959). Die Gestalt und das Sein. Gesammelte Abhandlungen über den Mythus und seine Bedeutungfür die Menschheit. Darmstadt: Wissenschaftliche Buchgesellschaft.

Poggi, S. (Ed.) (1989). Gestalt Psychology: Ist Origins, Foundations and Influence, An International Workshop, Firenze, 13-17 novembre.

Rohde, E. (1876), Der griechische Roman und Seine Vorläufer, Leipzig: Breitkopf \& Härtel.

Schlesier, R. (1994), Kulte, Kythen und Gelehrte. Anthropologie der Antike sei 1800, Frankfurt am Main: Fischer Taschenbuchverlag.

Spengler, O. (1923). Der Untergang des Abendlandes. München: Beck Verlag.

Vajda, L. (1973). Leo Frobenius heute. Conferencia en la Gesellschaft für Kulturmorphologie, 05.10.73.

Von Ehrenfels, C. (1890). Über Gestaltqualitäten. Vierteljahrsschrift für wissenschaftliche Philosophie, XIV(3), pp. 249-292.

Von Stietenkron, H.. Ed. (1986). Theologen und Theologien in verschiedenen Kulturkreisen. Düsseldorf: Patmos.

VV.AA. (1933). Leo Frobenius. Ein Lebenswerk aus der Zeit der Kulturwende dargestellt von seinen Freunden und Schülern. Leipzig: Koheler \& Amelang.

Wertheimer, M., (1912), Experimentelle Studien über das Sehen von Bewegung, Zeitschrift fur Psychologie, Band 61, pp.161-265. 